Check for updates

Cite this: RSC Adv., 2017, 7, 30214

Received 25th April 2017

Accepted 27th May 2017

DOI: 10.1039/c7ra04619g

rsc.li/rsc-advances

\title{
A palladium-catalyzed oxidative cross-coupling reaction between aryl pinacol boronates and $H$-phosphonates in ethanol $\dagger$
}

\author{
Te-Hsuan Chen, \$ Daggula Mallikarjuna Reddy $\$$ and Chin-Fa Lee (D) *
}

The first successful oxidative coupling reaction of aryl pinacol boronic esters with $\mathrm{H}$-phosphonates to deliver aryl phosphorous compounds is reported herein. These reactions between aryl boronic reagents and $\mathrm{H}$-phosphonates were carried out synergistically using a Pd catalyst, additive and oxidant. Without using bases and ligands, phosphorylation was accomplished in an environmentally-friendly manner under mild conditions in ethanol.

\section{Introduction}

The development of versatile methods for the synthesis of arylphosphorous compounds ${ }^{\mathbf{1}}$ has attracted much attention, owing to their broad applications in medicinal chemistry, ${ }^{2}$ materials chemistry, ${ }^{3}$ organic synthesis ${ }^{4}$ and catalysis. ${ }^{5}$ In 1981 , Hirao and co-workers reported the first construction of $\mathrm{C}\left(\mathrm{sp}^{2}\right)-\mathrm{P}$ bonds through palladium-catalyzed cross-coupling of aryl halides with $H$-phosphonates. ${ }^{6}$ During the past three decades, the scope of the reactions catalyzed by $\mathrm{Pd}, \mathrm{Cu}$ and $\mathrm{Ni}$ or other metals reagents to form $\mathrm{C}\left(\mathrm{sp}^{2}\right)-\mathrm{P}$ bonds employing functionalized arenes with phosphorus reagents has been significantly expanded to various aryl halides, ${ }^{7}$ triflates, ${ }^{8}$ imidazolylsulfonates, ${ }^{9}$ diazonium salts,${ }^{\mathbf{1 0}} \boldsymbol{o}$-aryl silyl triflates,${ }^{\mathbf{1 1}}$ arylhydrazines, ${ }^{\mathbf{1 2}}$ arylnitriles, ${ }^{13}$ aryl pivalates, ${ }^{14}$ sodium arylsulfinates ${ }^{15}$ and diaryliodonium salts. ${ }^{16}$ Arylphosphorus compounds can also be produced by the palladium or copper-catalyzed directed $\mathrm{C}-\mathrm{H} /$ $\mathrm{P}(\mathrm{O})-\mathrm{H}$ coupling reactions. ${ }^{17}$

Aryl boronic acids ${ }^{\mathbf{1 8}}$ are extensively used as substrates in transition-metal catalyzed cross-coupling reactions including Suzuki-Miyaura cross-coupling reactions, ${ }^{19} \mathrm{Cu}$-catalyzed $\mathrm{C}-\mathrm{O}$, $\mathrm{C}-\mathrm{N}, \mathrm{C}-\mathrm{S}$ and $\mathrm{C}-\mathrm{Se}$ coupling reactions, ${ }^{20}$ and Rh-catalyzed conjugate additions to carbonyl compounds, ${ }^{21}$ owing to their commercial availability and structural diversity. There are only a few examples reported for $\mathrm{C}-\mathrm{P}$ bond forming reactions using aryl boronic acids (Scheme 1a-c). ${ }^{22}$ Notably, additional ligands and bases are required in these works. Aryl boronic esters, especially aryl pinacol boronates, have received a great deal of attention in the catalysis community. In general, aryl boronic

Department of Chemistry, National Chung Hsing University, Taichung, Taiwan 402, Republic of China. E-mail: cfalee@dragon.nchu.edu.tw; Fax: +886-4-2286-2547; Tel: +886-4-2284-0411 ext. 810

$\uparrow$ Electronic supplementary information (ESI) available: For ${ }^{1} \mathrm{H}$ and ${ }^{13} \mathrm{C}$ spectra of componunds 3. See DOI: 10.1039/c7ra04619g

\$ Both authors contributed equally to this work. esters exhibit greater chemical stability and readily soluble in aprotic solvents. In most cases, they also exhibit stability towards column chromatography, which aids in their ease of isolation and purification. In addition, many are liquids at room temperature and can be easily distilled. However, to the best of our knowledge, the preparation of arylphosphorus compounds via oxidative cross coupling reactions of aryl boronic esters with $H$-phosphonates has never been developed. Herein, we report the first coupling reaction of aryl pinacol boronic esters with $H$-phosphonates through palladium catalysis (Scheme 1d). The features of this system include that the reactions are performed in ethanol and also declined the requirement of external ligand and base. The most important feature that we observed was that the reaction conditions used

(a) Larhed's work

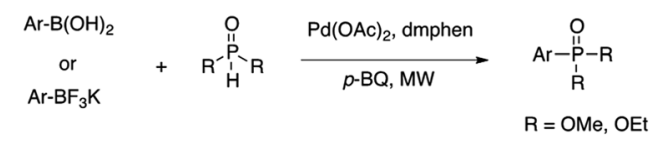

(b) Gao's work

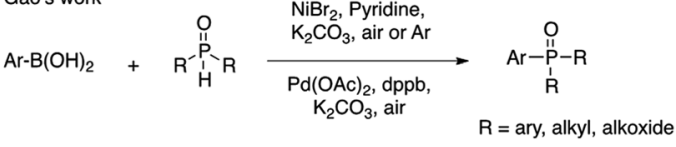

(c) Zhao's work

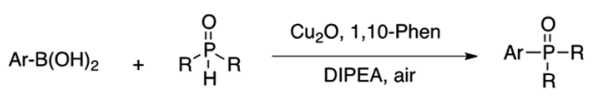$$
\mathrm{R}=\text { alkoxide }
$$

$$
\text { (d) This work: }
$$

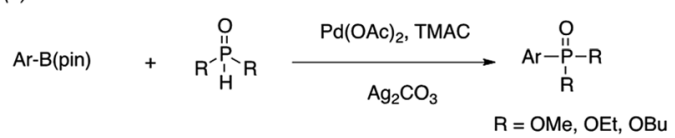

Scheme 1 Synthesis of aryl phosphorous compounds from arylboronic reagents. 
in Scheme 1a-c were not successful for the coupling of aryl pinacol boronate with $H$-phosphonate.

\section{Results and discussion}

We selected diethyl phosphite (1a) and phenyl pinacol boronate (2a) as model substrates to optimize the reaction conditions (Table 1). Initially, the reaction was carried out with $\mathrm{Pd}(\mathrm{OAc})_{2}(5$ mol\%) as the catalyst and $\mathrm{Ag}_{2} \mathrm{CO}_{3}$ (1.5 equiv.) as the oxidant in $\mathrm{DMF}$ at $80{ }^{\circ} \mathrm{C}$ for $24 \mathrm{~h}$; however, only a trace amount of the desired product diethyl phenylphosphonate 3aa was detected (entry 1). No product or only a trace amount of the product was observed when DMSO, $\mathrm{CH}_{3} \mathrm{CN}$ or toluene was used as the solvent (entries 2-4). To our delight, when the solvent was changed to ethanol, the coupling product 3aa was obtained at $22 \%$ yield (entry 5). Key role of ethanol in this coupling reaction to obtain the desired product can be explained by the possible coordination of ethanol at boron centre of aryl pinacol boronate. Due to the co-ordination of ethanol with aryl pinacol boronate the electron density at aryl system may be increased. When we chose tetramethylammonium bromide (TMAB), tetramethylammonium chloride (TMAC), $\mathrm{LiCl}, \mathrm{CsCl}$ and $\mathrm{TBACl}$ as the additives, the coupling product 3aa with TMAC and $\mathrm{LiCl}$ obtained $76 \%$ and $75 \%$ yields, respectively (entries 7 and 9), whereas other additives gave inferior results. To our delight, we chose TMAC as additive for this reaction. Enhancement of the

Table 1 Pd-catalyzed coupling of $\mathrm{PhB}$ (pin) with diethyl phosphite ${ }^{a}$

\begin{tabular}{|c|c|c|c|c|}
\hline & + & $\mathrm{Pd}(\mathrm{OAc})_{2}$ & $\underset{t}{\stackrel{\mathrm{ve}, \text { Oxidant }}{\longrightarrow}}$ & OEt \\
\hline Entry & Additive & Oxidant & Solvent & Yield $^{b}(\%)$ \\
\hline 1 & - & $\mathrm{Ag}_{2} \mathrm{CO}_{3}$ & $\mathrm{DMF}$ & Trace \\
\hline 2 & - & $\mathrm{Ag}_{2} \mathrm{CO}_{3}$ & DMSO & 0 \\
\hline 3 & - & $\mathrm{Ag}_{2} \mathrm{CO}_{3}$ & $\mathrm{CH}_{3} \mathrm{CN}$ & Trace \\
\hline 4 & - & $\mathrm{Ag}_{2} \mathrm{CO}_{3}$ & Toluene & Trace \\
\hline 5 & - & $\mathrm{Ag}_{2} \mathrm{CO}_{3}$ & EtOH & 22 \\
\hline 6 & TMAB & $\mathrm{Ag}_{2} \mathrm{CO}_{3}$ & EtOH & 25 \\
\hline 7 & TMAC & $\mathrm{Ag}_{2} \mathrm{CO}_{3}$ & EtOH & 76 \\
\hline 8 & $\mathrm{CsCl}$ & $\mathrm{Ag}_{2} \mathrm{CO}_{3}$ & EtOH & 50 \\
\hline 9 & $\mathrm{LiCl}$ & $\mathrm{Ag}_{2} \mathrm{CO}_{3}$ & EtOH & 75 \\
\hline 10 & TBAC & $\mathrm{Ag}_{2} \mathrm{CO}_{3}$ & EtOH & 35 \\
\hline 11 & TMAC & $\mathrm{Ag}_{2} \mathrm{CO}_{3}$ & $t-\mathrm{BuOH}$ & 32 \\
\hline 12 & TMAC & AgOAc & EtOH & 35 \\
\hline 13 & TMAC & $\mathrm{K}_{2} \mathrm{~S}_{2} \mathrm{O}_{8}$ & EtOH & N.R. \\
\hline 14 & TMAC & $\mathrm{Cu}(\mathrm{OAc})_{2}$ & EtOH & 28 \\
\hline 15 & TMAC & $\mathrm{O}_{2}$ & EtOH & N.R. \\
\hline $16^{c}$ & TMAC & $\mathrm{Ag}_{2} \mathrm{CO}_{3}$ & EtOH & 64 \\
\hline $17^{d}$ & TMAC & $\mathrm{Ag}_{2} \mathrm{CO}_{3}$ & EtOH & 62 \\
\hline $18^{e}$ & TMAC & $\mathrm{Ag}_{2} \mathrm{CO}_{3}$ & EtOH & 65 \\
\hline
\end{tabular}

${ }^{a}$ Reaction conditions: $1 \mathrm{a}(0.5 \mathrm{mmol}), 2 \mathrm{a}(1.5 \mathrm{eq}),. \mathrm{Pd}(\mathrm{OAc})_{2}(5 \mathrm{~mol} \%)$, additive (1.5 eq.), oxidant (1.5 eq.), and solvent $(3.0 \mathrm{~mL})$ under an atmosphere of $\mathrm{N}_{2}$ for $24 \mathrm{~h}$ at $80{ }^{\circ} \mathrm{C}$. ${ }^{b}$ Isolated yield. ${ }^{c} \mathrm{Pd}\left(\mathrm{PPh}_{3}\right)_{2} \mathrm{Cl}_{2}$ as the catalyst. ${ }^{d} \mathrm{Pd}\left(\mathrm{OCOCF}_{3}\right)_{2}$ as the catalyst. ${ }^{e} \mathrm{PdCl}_{2}$ as the catalyst. yield of product in the presence of TMAC was because of its phase transfer catalytic and inert counter ionic nature. The yield of the product was decreased to $32 \%$ when the reaction was conducted with tert-butanol as the solvent (entry 11). The choice of oxidant was crucial in this coupling reaction. When we replaced $\mathrm{Ag}_{2} \mathrm{CO}_{3}$ with $\mathrm{AgOAc}$ and $\mathrm{Cu}(\mathrm{OAc})_{2}$, both led to 3aa at lower yields (entries 12 and 14); meanwhile, no reaction occurred when $\mathrm{K}_{2} \mathrm{~S}_{2} \mathrm{O}_{8}$ or oxygen (ballon) was used as the oxidant (entries 13 and 15). The superiority of $\mathrm{Ag}_{2} \mathrm{CO}_{3}$ over other oxidants in this reaction could explained by its additional basic nature that can promote the deprotonation of $H$-phosphonates. In the screening of the palladium source, $\mathrm{Pd}\left(\mathrm{PPh}_{3}\right)_{2} \mathrm{Cl}_{2}$, $\mathrm{Pd}\left(\mathrm{OCOCF}_{3}\right)_{2}$ and $\mathrm{PdCl}_{2}$ showed lower reactivity (entries 16-18) compared with $\mathrm{Pd}(\mathrm{OAc})_{2}$.

We next investigated the relative reactivity of phenyl boronic acid derivatives (Table 2). When phenyl boronic acid (4) was used as a boron reagent, the coupling product 3aa was obtained at $61 \%$ yield. Phenyl boronic esters, such as phenyl hexylene glycol boronate (5) and phenyl neopentyl glycol boronate (6), were also tested, but both gave the product at lower yields when compared with phenyl pinacol boronate (2a).

With the optimized protocol from previous works, we examined the scope of the substrates in this catalytic system (Table 3). Various aryl pinacol boronates 2 were found to couple with 1a to produce the corresponding diethyl arylphosphonates 3 at moderate to good yields. It is worth mentioning that higher yields of products were obtained with aryl pinacol boronates 2 bearing electron-donating groups. Thus, with electron-donating substituents such as methyl and methoxy groups at the C-3 or C4 position of 2 , one could obtain the corresponding products at good yields (3aa-3ag). In contrast, substrates with electronwithdrawing trifluoromethyl and chloro groups led to products at lower yields (3ah and 3ai). When we used 3-bromophenyl

Table 2 Phosphorylation: relative reactivity of boron sources ${ }^{a}$

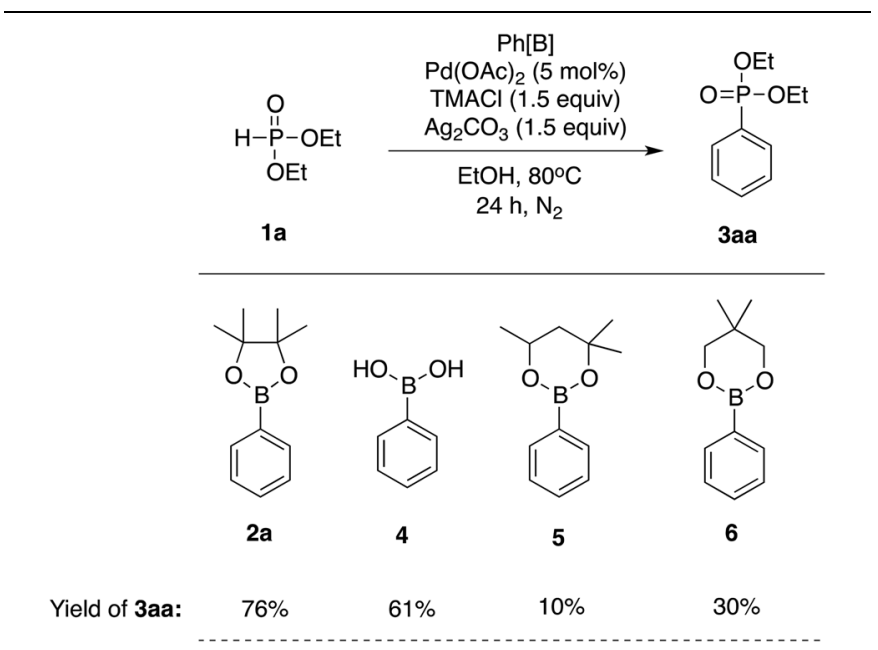

${ }^{a}$ Reaction conditions: $0.5 \mathrm{mmol}$ of $1 \mathrm{a}, 1.5$ equivalent of $\mathrm{Ph}[\mathrm{B}], 5 \mathrm{~mol} \%$ $\mathrm{Pd}(\mathrm{OAc})_{2}, 1.5$ equivalent of TMACl, 1.5 equivalent of $\mathrm{Ag}_{2} \mathrm{CO}_{3}$, and $3 \mathrm{~mL}$ of ethanol were charged into a Schlenk tube under nitrogen atmosphere and heated at $80^{\circ} \mathrm{C}$ for $24 \mathrm{~h}$. 
Table 3 Substrate scope of aryl pinacol boronates ${ }^{a}$

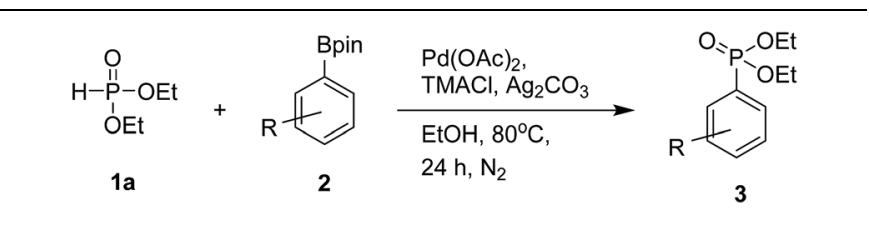

pinacol boronate as a coupling partner, the desired product was obtained in trace amount along with de-brominated arylphosphonate (3aa) in lower yield. Use of various aryl pinacol boronic esters such as 4-nitrophenyl, 4-methoxycarbonylphenyl, 4-cyanophenyl, naphthyl, 4-hydroxyphenyl pinacol boronates and heteroaryl pinacol boronates such as 3-thienyl, 5-indolyl pinacol boronates as substrates to couple with $H$-phosphonate, failed to the formation of desired products instead detected the deborolyted products. Furthermore, we also tested the reaction by using ortho substituted aryl pinacol boronates such as 2-chlorophenyl and mesityl pinacol boronates. Under the reaction conditions, these two substrates also failed to react with $H$-phosphonate. The formation of de-borolyted product was detected by GC analysis. Another important factor that was responsible for lowering the yield of product was formation trialkylphosphates by the reaction of $H$-phosphonate with solvent (ethanol).

The generality of this coupling reaction was further demonstrated by using two other substituted phosphites, dimethyl phosphite (1b) and dibutyl phosphite (1c). When screening with different aryl pinacol boronates 2 , both phosphites could produce the corresponding products at moderate to good yields (Table 4 ).

Although the mechanism of this tranformation is not clear at this stage, based on experimental results, we propose that
Table 4 Pd-catalyzed coupling of aryl pinacol boronates 2 with phosphites $^{a}$
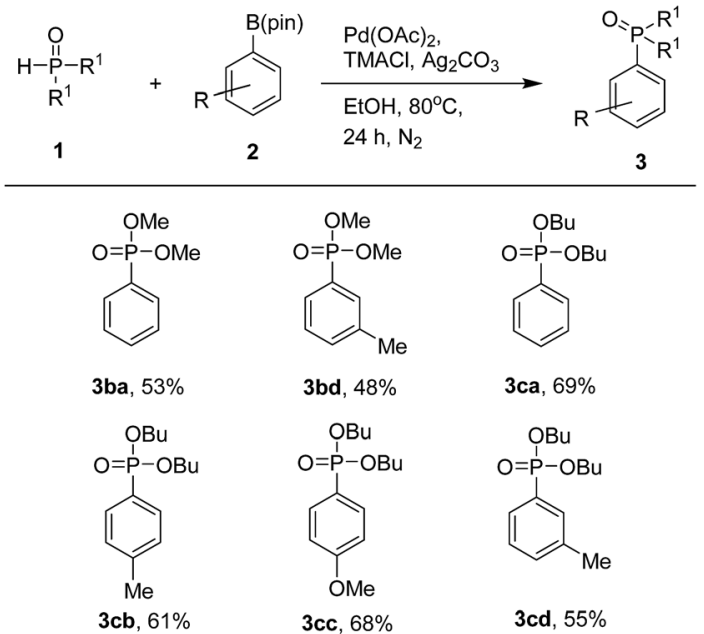

3ca, $69 \%$
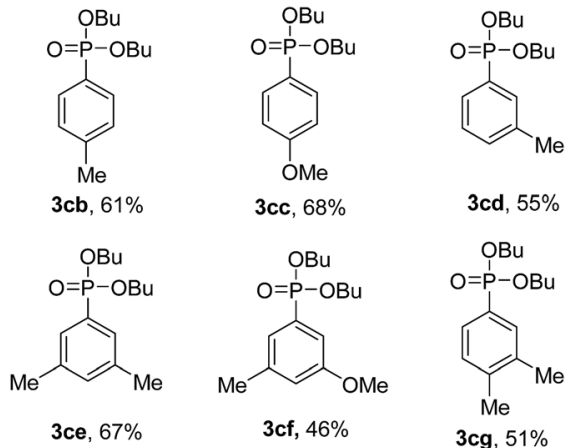

3cd, $55 \%$

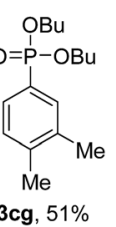

${ }^{a}$ Reaction conditions: $0.5 \mathrm{mmol}$ of $1,1.5$ equivalent of boronate 2,5 mol\% $\mathrm{Pd}(\mathrm{OAc})_{2}, 1.5$ equivalent of TMACl, 1.5 equivalent of $\mathrm{Ag}_{2} \mathrm{CO}_{3}$, and $3 \mathrm{~mL}$ of ethanol were charged into a Schlenk tube under nitrogen atmosphere and heated at $80^{\circ} \mathrm{C}$ for $24 \mathrm{~h}$.

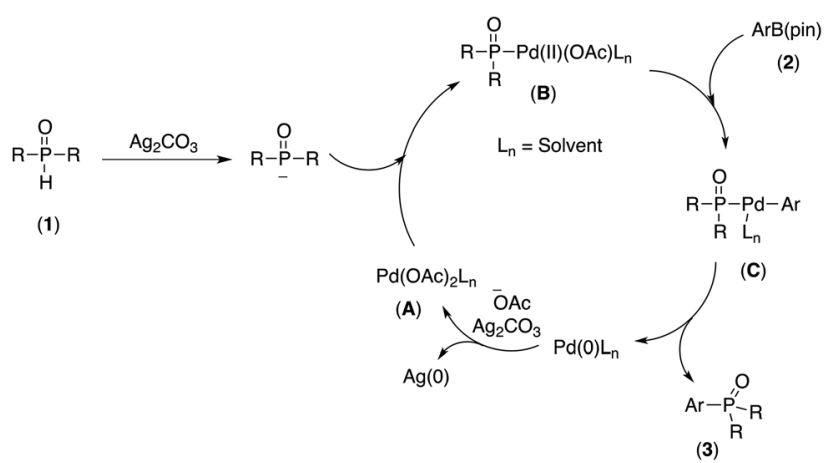

Scheme 2 Plausible mechanism for the formation of 3.

this Pd-catalyzed cross-coupling takes place via the catalytic cycle shown in Scheme 2. Firstly, the Pd(II) complex A reacted with the phosphorous nucleophile generated by deprotonation of the $\mathrm{P}(\mathrm{O}) \mathrm{H}$ compound in the presence of $\mathrm{a}_{2} \mathrm{Ag}_{3}$ to provide intermediate $\mathbf{B} .^{22 a, d}$ The resulting intermediate $\mathbf{B}$ associated with arylpinacol boronate (2) to give another intermediate $\mathbf{C ,}^{\mathbf{2 2 a}, \boldsymbol{d}, \mathbf{2 3}}$ which upon reductive elimination, afforded the desired coupling product (3) and $\operatorname{Pd}(0)$ species. Finally, the $\mathrm{Pd}(0)$ species was oxidized by $\mathrm{Ag}_{2} \mathrm{CO}_{3}$, leading to the regeneration of $\mathrm{Pd}(\mathrm{II})$ complex $\mathbf{A}$ as a catalytically active species. 


\section{Conclusion}

In conclusion, we demonstrated a novel Pd-catalyzed crosscoupling reaction of aryl pinacol boronates with $H$-phosphonates, which afforded good yields of arylphosphorus compounds under mild conditions in ethanol. A variety of readily available aryl pinacol boronates can be used in this coupling reaction. We believe that this is a new protocol for the construction of valuable $\mathrm{C}-\mathrm{P}$ bonds from readily available, environmentally friendly chemical sources.

\section{Experimental}

\section{General information}

All commercial chemicals were used as received except where noted. Aryl pinacol boronates were all prepared through literature procedures. ${ }^{24,25}$ Experiments were performed under a dinitrogen atmosphere using standard Schlenk techniques unless otherwise stated. Flash chromatography was performed on Merck silica gel 60 (230-400 mesh).

\section{Analysis}

NMR spectra were recorded on a Varian Unity Inova-600 or a Varian Mercury-400 instrument using $\mathrm{CDCl}_{3}$ as a solvent. Chemical shifts are reported in parts per million (ppm) and referenced to the residual solvent resonance. Coupling constant $(J)$ are reported in hertz $(\mathrm{Hz})$. Standard abbreviations indicating multiplicity were used as follows: $\mathrm{s}=$ singlet, $\mathrm{d}=$ doublet, $\mathrm{t}=$ triplet, $\mathrm{dd}=$ double doublet, $\mathrm{q}=$ quartet, quin $=$ quintet, $\mathrm{m}=$ multiplet, $\mathrm{b}=$ broad. GC-MS analysis were performed on a Agilent Technologies 5977A GC equipped with Agilent 7890B MS. High-resolution mass spectra were carried out on a Jeol JMS-HX 110 spectrometer by the services at the National Chung Hsing University.

\section{General procedure for synthesis of compound 3}

To a mixture of aryl pinacol boronic ester $(0.75 \mathrm{mmol}, 1.5$ equiv.), $\mathrm{Pd}(\mathrm{OAc})_{2}$ (5.6 mg, $\left.5 \mathrm{~mol} \%\right), \mathrm{Ag}_{2} \mathrm{CO}_{3}(0.21 \mathrm{~g}, 1.5$ equiv.) and tetramethylammonium chloride $(82 \mathrm{mg}, 1.5$ equiv.) were added $H$-phosphonate $(0.5 \mathrm{mmol})$ and ethanol $(3.0 \mathrm{~mL})$ under nitrogen atmosphere. The resulting suspension was heated at $80^{\circ} \mathrm{C}$ for $24 \mathrm{~h}$. After $24 \mathrm{~h}$, the reaction mixture was cooled down to room temperature and diluted with ethyl acetate $(20 \mathrm{~mL})$. The resulting solution was directly filtered through a pad of celite and the filtrate was concentrated under vacuum. The crude product was purified by flash column chromatography (ethyl acetate $/$ hexane $=30-80 / 20-70$ ).

Diethyl phenylphosphonate (3aa): ${ }^{22}$ Following the general procedure for compound 3 , using phenylboronic acid pinacol ester $2 \mathrm{a}(153 \mathrm{mg}, 0.75 \mathrm{mmol}), \mathrm{Pd}(\mathrm{OAc})_{2}(5.6 \mathrm{mg}, 5 \mathrm{~mol} \%)$, $\mathrm{Ag}_{2} \mathrm{CO}_{3}(0.21 \mathrm{~g}, 0.75 \mathrm{mmol})$ and tetramethylammonium chloride ( $82 \mathrm{mg}, 0.75 \mathrm{mmol})$ were added diethyl phosphite $1 \mathrm{a}(67.5$ $\mu \mathrm{L}, 0.50 \mathrm{mmol})$ in ethanol $(3.0 \mathrm{~mL})$, then purified by column chromatography $\left(\mathrm{SiO}_{2}\right.$, ethyl acetate/hexane $\left.=30-80 \%\right)$ to provide 3aa as a colorless oil ( $81.3 \mathrm{mg}, 76 \%$ yield). ${ }^{1} \mathrm{H}$ NMR (400 $\left.\mathrm{MHz}, \mathrm{CDCl}_{3}\right): \delta 7.79-7.85(\mathrm{~m}, 2 \mathrm{H}), 7.54-7.58(\mathrm{~m}, 1 \mathrm{H}), 7.45-7.50$ (m, 2H), 4.05-4.19 (m, 4H), $1.33(\mathrm{t}, J=7.2 \mathrm{~Hz}, 3 \mathrm{H}), 1.32(\mathrm{t}, J=$ $7.2 \mathrm{~Hz}, 3 \mathrm{H}) ;{ }^{13} \mathrm{C}$ NMR $\left(100 \mathrm{MHz}, \mathrm{CDCl}_{3}\right): \delta 132.3\left(\mathrm{~d}, J_{\mathrm{C}-\mathrm{P}}=2.7\right.$ $\mathrm{Hz}), 131.7\left(\mathrm{~d}, J_{\mathrm{C}-\mathrm{P}}=9.9 \mathrm{~Hz}\right), 128.4\left(\mathrm{~d}, J_{\mathrm{C}-\mathrm{P}}=14.8 \mathrm{~Hz}\right), 128.2(\mathrm{~d}$, $\left.J_{\mathrm{C}-\mathrm{P}}=186.8 \mathrm{~Hz}\right), 62.0\left(\mathrm{~d}, J_{\mathrm{C}-\mathrm{P}}=5.3 \mathrm{~Hz}\right), 16.2\left(\left(\mathrm{~d}, J_{\mathrm{C}-\mathrm{P}}=6.4 \mathrm{~Hz}\right)\right)$; ${ }^{31} \mathrm{P}$ NMR (162 MHz, $\left.\mathrm{CDCl}_{3}\right): \delta 19.54$.

Diethyl 4-methylphenylphosphonate (3ab):. ${ }^{22}$ Following the general procedure for compound 3, using 4-methylphenylboronic acid pinacol ester (163.6 mg, $0.75 \mathrm{mmol}), \mathrm{Pd}(\mathrm{OAc})_{2}(5.6 \mathrm{mg}$, $5 \mathrm{~mol} \%), \mathrm{Ag}_{2} \mathrm{CO}_{3}(0.21 \mathrm{~g}, 0.75 \mathrm{mmol})$ and tetramethylammonium chloride ( $82 \mathrm{mg}, 0.75 \mathrm{mmol}$ ) were added diethyl phosphite $1 \mathrm{a}(67.5 \mu \mathrm{L}, 0.50 \mathrm{mmol})$ in ethanol $(3.0 \mathrm{~mL})$, then purified by column chromatography $\left(\mathrm{SiO}_{2}\right.$, ethyl acetate/hexane $=30-$ $80 \%)$ to provide $3 \mathrm{ab}$ as a colorless oil $\left(66.1 \mathrm{mg}, 58 \%\right.$ yield). ${ }^{1} \mathrm{H}$ NMR (400 MHz, $\left.\mathrm{CDCl}_{3}\right): \delta 7.72(\mathrm{~d}, J=8.0 \mathrm{~Hz}, 1 \mathrm{H}), 7.69(\mathrm{~d}, J=$ $8.0 \mathrm{~Hz}, 1 \mathrm{H}), 7.26-7.29(\mathrm{~m}, 2 \mathrm{H}), 4.04-4.15(\mathrm{~m}, 4 \mathrm{H}), 2.41(\mathrm{~s}, 3 \mathrm{H})$, $1.32(\mathrm{t}, J=7.2 \mathrm{~Hz}, 6 \mathrm{H}) ;{ }^{13} \mathrm{C} \mathrm{NMR}\left(100 \mathrm{MHz}, \mathrm{CDCl}_{3}\right): \delta 142.92(\mathrm{~d}$, $\left.J_{\mathrm{C}-\mathrm{P}}=3.1 \mathrm{~Hz}\right), 131.8\left(\mathrm{~d}, J_{\mathrm{C}-\mathrm{P}}=10.2 \mathrm{~Hz}\right), 129.2\left(\mathrm{~d}, J_{\mathrm{C}-\mathrm{P}}=15.5 \mathrm{~Hz}\right)$, $124.8\left(\mathrm{~d}, J_{\mathrm{C}-\mathrm{P}}=189.0 \mathrm{~Hz}\right), 61.94\left(\mathrm{~d}, J_{\mathrm{C}-\mathrm{P}}=5.3 \mathrm{~Hz}\right), 21.6\left(\mathrm{~d}, J_{\mathrm{C}-\mathrm{P}}=\right.$ $1.2 \mathrm{~Hz}), 16.26$ (d, $\left.J_{\mathrm{C}-\mathrm{P}}=6.5 \mathrm{~Hz}\right) ;{ }^{31} \mathrm{P}$ NMR (162 MHz, $\left.\mathrm{CDCl}_{3}\right)$ : $\delta 20.21$.

Diethyl 4-methoxyphenylphosphonate (3ac):. ${ }^{22}$ Following the general procedure for compound 3, using 4-methoxyphenylboronic acid pinacol ester (175.6 $\mathrm{mg}, 0.75 \mathrm{mmol}$ ), $\mathrm{Pd}(\mathrm{OAc})_{2}$ (5.6 mg, $\left.5 \mathrm{~mol} \%\right), \mathrm{Ag}_{2} \mathrm{CO}_{3}(0.21 \mathrm{~g}, 0.75 \mathrm{mmol})$ and tetramethylammonium chloride $(82 \mathrm{mg}, 0.75 \mathrm{mmol})$ were added diethyl phosphite $1 \mathrm{a}(67.5 \mu \mathrm{L}, 0.50 \mathrm{mmol})$ in ethanol (3.0 $\mathrm{mL})$, then purified by column chromatography $\left(\mathrm{SiO}_{2}\right.$, ethyl acetate $/$ hexane $=30-80 \%)$ to provide 3ac as a colorless oil (80.5 mg, 66\% yield). ${ }^{1} \mathrm{H}$ NMR (400 $\left.\mathrm{MHz}, \mathrm{CDCl}_{3}\right): \delta 7.77$ (d, $J=$ $8.8 \mathrm{~Hz}, 1 \mathrm{H}), 7.73$ (d, $J=8.8,1 \mathrm{H}), 6.95-6.99(\mathrm{~m}, 2 \mathrm{H}), 4.01-4.16$ $(\mathrm{m}, 4 \mathrm{H}), 3.86(\mathrm{~s}, 3 \mathrm{H}), 1.31(\mathrm{t}, J=7.2 \mathrm{~Hz}, 6 \mathrm{H}) ;{ }^{13} \mathrm{C} \mathrm{NMR}(100 \mathrm{MHz}$, $\left.\mathrm{CDCl}_{3}\right): \delta 162.8\left(\mathrm{~d}, J_{\mathrm{C}-\mathrm{P}}=3 \mathrm{~Hz}\right), 133.8\left(\mathrm{~d}, J_{\mathrm{C}-\mathrm{P}}=11.3 \mathrm{~Hz}\right), 119.4$ $\left(\mathrm{d}, J_{\mathrm{C}-\mathrm{P}}=193.5 \mathrm{~Hz}\right), 114.0\left(\mathrm{~d}, J_{\mathrm{C}-\mathrm{P}}=15.9 \mathrm{~Hz}\right), 61.9\left(\mathrm{~d}, J_{\mathrm{C}-\mathrm{P}}=4.9\right.$ $\mathrm{Hz}), 55.3,16.3\left(\mathrm{~d}, J_{\mathrm{C}-\mathrm{P}}=6.4 \mathrm{~Hz}\right) ;{ }^{31} \mathrm{P}$ NMR $\left(162 \mathrm{MHz}, \mathrm{CDCl}_{3}\right)$ : $\delta 20.78$.

Diethyl 3-methylphenylphosphonate (3ad):. ${ }^{22}$ Following the general procedure for compound 3, using 3-methylphenylboronic acid pinacol ester (163.6 mg, $0.75 \mathrm{mmol}), \mathrm{Pd}(\mathrm{OAc})_{2}(5.6 \mathrm{mg}$, $5 \mathrm{~mol} \%), \mathrm{Ag}_{2} \mathrm{CO}_{3}(0.21 \mathrm{~g}, 0.75 \mathrm{mmol})$ and tetramethylammonium chloride ( $82 \mathrm{mg}, 0.75 \mathrm{mmol}$ ) were added diethyl phosphite $1 \mathrm{a}(67.5 \mu \mathrm{L}, 0.50 \mathrm{mmol})$ in ethanol $(3.0 \mathrm{~mL})$, then purified by column chromatography $\left(\mathrm{SiO}_{2}\right.$, ethyl acetate/hexane $=30-$ $80 \%)$ to provide 3 ad as a colorless oil ( $84.4 \mathrm{mg}, 74 \%$ yield). ${ }^{1} \mathrm{H}$ NMR (400 MHz, $\left.\mathrm{CDCl}_{3}\right): \delta 7.57-7.66(\mathrm{~m}, 2 \mathrm{H}), 7.30-7.37(\mathrm{~m}, 2 \mathrm{H})$, 4.04-4.18 (m, 4H), 2.40 (s, 3H), 1.33 (t, $J=7.2 \mathrm{~Hz}, 6 \mathrm{H}) ;{ }^{13} \mathrm{C} \mathrm{NMR}$ $\left(100 \mathrm{MHz}, \mathrm{CDCl}_{3}\right): \delta 138.2\left(\mathrm{~d}, J_{\mathrm{C}-\mathrm{P}}=14.8 \mathrm{~Hz}\right), 133.08\left(\mathrm{~d}, J_{\mathrm{C}-\mathrm{P}}=\right.$ $3.1 \mathrm{~Hz}), 132.1\left(\mathrm{~d}, J_{\mathrm{C}-\mathrm{P}}=9.8 \mathrm{~Hz}\right), 128.5\left(\mathrm{~d}, J_{\mathrm{C}-\mathrm{P}}=34.2 \mathrm{~Hz}\right), 128.4$ $\left(\mathrm{d}, J_{\mathrm{C}-\mathrm{P}}=40.2 \mathrm{~Hz}\right), 127.8\left(\mathrm{~d}, J_{\mathrm{C}-\mathrm{P}}=185.9 \mathrm{~Hz}\right), 61.9\left(\mathrm{~d}, J_{\mathrm{C}-\mathrm{P}}=5.3\right.$ $\mathrm{Hz}), 21.2,16.2\left(\mathrm{~d}, J_{\mathrm{C}-\mathrm{P}}=6.5 \mathrm{~Hz}\right) ;{ }^{31} \mathrm{P}$ NMR (162 MHz, $\left.\mathrm{CDCl}_{3}\right)$ : $\delta 19.91$.

Diethyl 3,5-dimethylphenylphosphonate (3ae):. ${ }^{12}$ Following the general procedure for compound 3, using 3,5-dimethylphenylboronic acid pinacol ester (174.1 $\mathrm{mg}, 0.75 \mathrm{mmol}$ ), $\mathrm{Pd}(\mathrm{OAc})_{2}$ (5.6 mg, $\left.5 \mathrm{~mol} \%\right), \mathrm{Ag}_{2} \mathrm{CO}_{3}(0.21 \mathrm{~g}, 0.75 \mathrm{mmol})$ and tetramethylammonium chloride $(82 \mathrm{mg}, 0.75 \mathrm{mmol})$ were added diethyl phosphite $1 \mathrm{a}(67.5 \mu \mathrm{L}, 0.50 \mathrm{mmol})$ in ethanol $(3.0$ $\mathrm{mL})$, then purified by column chromatography $\left(\mathrm{SiO}_{2}\right.$, ethyl 
acetate/hexane $=30-80 \%$ ) to provide 3 ae as a colorless oil (107.7 mg, 89\% yield). ${ }^{1} \mathrm{H}$ NMR (400 MHz, $\left.\mathrm{CDCl}_{3}\right): \delta 7.44(\mathrm{~s}, 1 \mathrm{H})$, $7.41(\mathrm{~s}, 1 \mathrm{H}), 7.18(\mathrm{~s}, 1 \mathrm{H}), 4.03-4.18(\mathrm{~m}, 4 \mathrm{H}), 2.36(\mathrm{~s}, 6 \mathrm{H}), 1.33^{*}(\mathrm{t}$, $J=7.2 \mathrm{~Hz}, 3 \mathrm{H}), 1.32 *(\mathrm{t}, J=7.2 \mathrm{~Hz}, 3 \mathrm{H})$; *This triplet was merged with other triplet; ${ }^{13} \mathrm{C}$ NMR $\left(100 \mathrm{MHz} \mathrm{CDCl}_{3}\right): \delta 138.1$ $\left(\mathrm{d}, J_{\mathrm{C}-\mathrm{P}}=15.6 \mathrm{~Hz}\right), 134.1\left(\mathrm{~d}, J_{\mathrm{C}-\mathrm{P}}=3.0 \mathrm{~Hz}\right), 129.3\left(\mathrm{~d}, J_{\mathrm{C}-\mathrm{P}}=9.9\right.$ $\mathrm{Hz}), 127.6\left(\mathrm{~d}, J_{\mathrm{C}-\mathrm{P}}=185.2 \mathrm{~Hz}\right), 61.9\left(\mathrm{~d}, J_{\mathrm{C}-\mathrm{P}}=5.3 \mathrm{~Hz}\right), 21.1\left(\mathrm{~d}, J_{\mathrm{C}-}\right.$ $\mathrm{p}=1.1 \mathrm{~Hz}), 16.2\left(\mathrm{~d}, J_{\mathrm{C}-\mathrm{P}}=6.8 \mathrm{~Hz}\right) ;{ }^{31} \mathrm{P} \mathrm{NMR}\left(162 \mathrm{MHz}, \mathrm{CDCl}_{3}\right)$ : $\delta 20.48$.

Diethyl (3-methoxy-5-methylphenyl)phosphonate (3af). Following the general procedure for compound 3, using (3methoxy-5-methyl)phenyl boronic acid pinacol ester $(186.1 \mathrm{mg}$, $0.75 \mathrm{mmol}$ ), $\mathrm{Pd}(\mathrm{OAc})_{2}(5.6 \mathrm{mg}, 5 \mathrm{~mol} \%), \mathrm{Ag}_{2} \mathrm{CO}_{3}(0.21 \mathrm{~g}, 0.75$ $\mathrm{mmol}$ ) and tetramethylammonium chloride $(82 \mathrm{mg}, 0.75 \mathrm{mmol})$ were added diethyl phosphite 1a $(67.5 \mu \mathrm{L}, 0.50 \mathrm{mmol})$ in ethanol $(3.0 \mathrm{~mL})$, then purified by column chromatography $\left(\mathrm{SiO}_{2}\right.$, ethyl acetate/hexane $\left.=30-80 \%\right)$ to provide 3af as a colorless oil ( $87.8 \mathrm{mg}, 68 \%$ yield). ${ }^{1} \mathrm{H}$ NMR (400 $\left.\mathrm{MHz}, \mathrm{CDCl}_{3}\right)$ : $\delta 7.21(\mathrm{~d}, J=13.6 \mathrm{~Hz}, 1 \mathrm{H}), 7.13(\mathrm{~d}, J=14.8 \mathrm{~Hz}, 1 \mathrm{H}), 6.90(\mathrm{~s}, 1 \mathrm{H})$, 4.18-4.04 (m, 4H), $3.82(\mathrm{~s}, 3 \mathrm{H}), 2.37$ (s, 3H), 1.33 (t, $J=7.2 \mathrm{~Hz}$, $6 \mathrm{H}) ;{ }^{13} \mathrm{C} \mathrm{NMR}\left(100 \mathrm{MHz}, \mathrm{CDCl}_{3}\right): \delta 159.3\left(\mathrm{~d}, J_{\mathrm{C}-\mathrm{P}}=20.1 \mathrm{~Hz}\right)$, $140.0\left(\mathrm{~d}, J_{\mathrm{C}-\mathrm{P}}=17.9 \mathrm{~Hz}\right), 128.9\left(\mathrm{~d}, J_{\mathrm{C}-\mathrm{P}}=185.2 \mathrm{~Hz}\right), 124.7\left(\mathrm{~d}, J_{\mathrm{C}-\mathrm{P}}\right.$ $=9.5 \mathrm{~Hz}), 119.4\left(\mathrm{~d}, J_{\mathrm{C}-\mathrm{P}}=3.0 \mathrm{~Hz}\right), 113.2\left(\mathrm{~d}, J_{\mathrm{C}-\mathrm{P}}=11.4 \mathrm{~Hz}\right), 62.0$ $\left(\mathrm{d}, J_{\mathrm{C}-\mathrm{P}}=4.9 \mathrm{~Hz}\right), 55.3,21.3,16.24\left(\mathrm{~d}, J_{\mathrm{C}-\mathrm{P}}=6.5 \mathrm{~Hz}\right) ;{ }^{31} \mathrm{P}$ NMR $\left(162 \mathrm{MHz}, \mathrm{CDCl}_{3}\right): \delta$ 19.98; HRMS-EI calcd for $\mathrm{C}_{12} \mathrm{H}_{19} \mathrm{O}_{4} \mathrm{P}[\mathrm{M}]^{+}$: 258.1021, found: 258.1028.

Diethyl 3,4-dimethylphenylphosphonate (3ag): ${ }^{11}$ Following the general procedure for compound 3, using 3,4-dimethylphenylboronic acid pinacol ester (174.1 $\mathrm{mg}, 0.75 \mathrm{mmol}$ ), $\mathrm{Pd}(\mathrm{OAc})_{2}$ (5.6 mg, $\left.5 \mathrm{~mol} \%\right), \mathrm{Ag}_{2} \mathrm{CO}_{3}(0.21 \mathrm{~g}, 0.75 \mathrm{mmol})$ and tetramethylammonium chloride $(82 \mathrm{mg}, 0.75 \mathrm{mmol})$ were added diethyl phosphite $1 \mathrm{a}(67.5 \mu \mathrm{L}, 0.50 \mathrm{mmol})$ in ethanol (3.0 $\mathrm{mL})$, then purified by column chromatography $\left(\mathrm{SiO}_{2}\right.$, ethyl acetate/hexane $=30-80 \%)$ to provide $3 a g$ as a colorless oil (87.2 mg, 72\% yield). ${ }^{1} \mathrm{H}$ NMR (400 MHz, $\left.\mathrm{CDCl}_{3}\right): \delta 7.50-7.61(\mathrm{~m}$, $2 \mathrm{H}), 7.21-7.28(\mathrm{~m}, 1 \mathrm{H}), 4.02-4.17(\mathrm{~m} 4 \mathrm{H}), 2.31(\mathrm{~s}, 6 \mathrm{H}), 1.33(\mathrm{t}, J=$ $7.2 \mathrm{~Hz}, 3 \mathrm{H}), 1.32(\mathrm{t}, J=7.2 \mathrm{~Hz}, 3 \mathrm{H}) ;{ }^{13} \mathrm{C} \mathrm{NMR}\left(100 \mathrm{MHz}, \mathrm{CDCl}_{3}\right)$ : $\delta 141.7\left(\mathrm{~d}, J_{\mathrm{C}-\mathrm{P}}=3.4 \mathrm{~Hz}\right), 137.0\left(\mathrm{~d}, J_{\mathrm{C}-\mathrm{P}}=15.2 \mathrm{~Hz}\right), 132.8\left(\mathrm{~d}, J_{\mathrm{C}-\mathrm{P}}\right.$ $=10.3 \mathrm{~Hz}), 129.6\left(\mathrm{~d}, J_{\mathrm{C}-\mathrm{P}}=45.9 \mathrm{~Hz}\right), 129.5\left(\mathrm{~d}, J_{\mathrm{C}-\mathrm{P}}=40.2 \mathrm{~Hz}\right)$, $125.1\left(\mathrm{~d}, J_{\mathrm{C}-\mathrm{P}}=187.8 \mathrm{~Hz}\right), 61.9\left(\mathrm{~d}, J_{\mathrm{C}-\mathrm{P}}=5.3 \mathrm{~Hz}\right), 19.9,19.6,16.3$ $\left(\mathrm{d}, J_{\mathrm{C}-\mathrm{P}}=6.8 \mathrm{~Hz}\right) ;{ }^{31} \mathrm{P} \mathrm{NMR}\left(162 \mathrm{MHz}, \mathrm{CDCl}_{3}\right): \delta 20.59$.

Diethyl 4-trifluoromethylmethylphenylphosphonate (3ah):.22 Following the general procedure for compound 3, using 4-trifluorophenylboronic acid pinacol ester $(204.1 \mathrm{mg}, 0.75 \mathrm{mmol})$, $\mathrm{Pd}(\mathrm{OAc})_{2}$ (5.6 mg, $\left.5 \mathrm{~mol} \%\right), \mathrm{Ag}_{2} \mathrm{CO}_{3}(0.21 \mathrm{~g}, 0.75 \mathrm{mmol})$ and tetramethylammonium chloride $(82 \mathrm{mg}, 0.75 \mathrm{mmol})$ were added diethyl phosphite $1 \mathrm{a}(67.5 \mu \mathrm{L}, 0.50 \mathrm{mmol})$ in ethanol (3.0 $\mathrm{mL})$, then purified by column chromatography $\left(\mathrm{SiO}_{2}\right.$, ethyl acetate/hexane $=30-80 \%)$ to provide $3 \mathbf{a h}$ as a colorless oil (25.4 mg, 18\% yield). ${ }^{1} \mathrm{H}$ NMR (400 $\mathrm{MHz}, \mathrm{CDCl}_{3}$ ): $\delta 7.97$ (d, $J=$ $7.6 \mathrm{~Hz}, 1 \mathrm{H}), 7.94$ (d, $J=8.0 \mathrm{~Hz}, 1 \mathrm{H}), 7.72-7.75$ (m, 2H), 4.10-4.20 $(\mathrm{m}, 4 \mathrm{H}), 1.34(\mathrm{t}, J=6.8 \mathrm{~Hz}, 6 \mathrm{H}) ;{ }^{13} \mathrm{C} \mathrm{NMR}\left(100 \mathrm{MHz}, \mathrm{CDCl}_{3}\right)$ : $\delta 134.0(\mathrm{~d}, J=33.0 \mathrm{~Hz}), 132.7(\mathrm{~d}, J=185.5 \mathrm{~Hz}), 132.2(\mathrm{~d}, J=9.9$ $\mathrm{Hz}), 125.3(\mathrm{dq}, J=15.1,3.8 \mathrm{~Hz}), 123.5(\mathrm{q}, J=270.6 \mathrm{~Hz}), 62.5(\mathrm{~d}, J$ $=5.7 \mathrm{~Hz}), 16.3(\mathrm{~d}, J=6.5 \mathrm{~Hz}) ;{ }^{31} \mathrm{P} \mathrm{NMR}\left(162 \mathrm{MHz}, \mathrm{CDCl}_{3}\right)$ : $\delta 17.02 ;{ }^{19} \mathrm{~F}$ NMR $\left(376 \mathrm{MHz}, \mathrm{CDCl}_{3}\right): \delta-63.3$.
Diethyl 4-chlorophenylphosphonate (3ai):. ${ }^{12}$ Following the general procedure for compound 3, using 4-chlorophenylboronic acid pinacol ester (178.6 mg, $0.75 \mathrm{mmol}), \mathrm{Pd}(\mathrm{OAc})_{2}(5.6 \mathrm{mg}$, $5 \mathrm{~mol} \%), \mathrm{Ag}_{2} \mathrm{CO}_{3}(0.21 \mathrm{~g}, 0.75 \mathrm{mmol})$ and tetramethylammonium chloride (82 $\mathrm{mg}, 0.75 \mathrm{mmol}$ ) were added diethyl phosphite 1a $(67.5 \mu \mathrm{L}, 0.50 \mathrm{mmol})$ in ethanol $(3.0 \mathrm{~mL})$, then purified by column chromatography $\left(\mathrm{SiO}_{2}\right.$, ethyl acetate/hexane $=30-$ $80 \%)$ to provide 3 ai as a colorless oil (33.5 mg, $27 \%$ yield). ${ }^{1} \mathrm{H}$ NMR (400 MHz, $\left.\mathrm{CDCl}_{3}\right): \delta 7.77(\mathrm{~d}, J=8.4 \mathrm{~Hz}, 1 \mathrm{H}), 7.73(\mathrm{~d}, J=$ $8.8 \mathrm{~Hz}, 1 \mathrm{H}), 7.44-7.47(\mathrm{~m}, 2 \mathrm{H}), 4.04-4.19(\mathrm{~m}, 4 \mathrm{H}), 1.33(\mathrm{t}, J=$ $7.2 \mathrm{~Hz}, 6 \mathrm{H}) ;{ }^{13} \mathrm{C}$ NMR $\left(100 \mathrm{MHz}, \mathrm{CDCl}_{3}\right): \delta 138.9\left(\mathrm{~d}, J_{\mathrm{C}-\mathrm{P}}=3.8\right.$ $\mathrm{Hz}), 133.2\left(\mathrm{~d}, J_{\mathrm{C}-\mathrm{P}}=10.7 \mathrm{~Hz}\right), 128.8\left(\mathrm{~d}, J_{\mathrm{C}-\mathrm{P}}=15.6 \mathrm{~Hz}\right), 126.9(\mathrm{~d}$, $\left.J_{\mathrm{C}-\mathrm{P}}=190.1 \mathrm{~Hz}\right), 62.25\left(\mathrm{~d}, J_{\mathrm{C}-\mathrm{P}}=5.7 \mathrm{~Hz}\right), 16.3\left(\mathrm{~d}, J_{\mathrm{C}-\mathrm{P}}=6.4 \mathrm{~Hz}\right)$; ${ }^{31} \mathrm{P}$ NMR (162 MHz, $\left.\mathrm{CDCl}_{3}\right): \delta 18.67$.

Dimethyl phenylphosphonate (3ba):. ${ }^{22}$ Following the general procedure for compound 3 , using phenylboronic acid pinacol ester (153 mg, $0.75 \mathrm{mmol}$ ), $\mathrm{Pd}(\mathrm{OAc})_{2}$ (5.6 mg, $\left.5 \mathrm{~mol} \%\right), \mathrm{Ag}_{2} \mathrm{CO}_{3}$ $(0.21 \mathrm{~g}, \quad 0.75 \mathrm{mmol})$ and tetramethylammonium chloride (82 $\mathrm{mg}, 0.75 \mathrm{mmol}$ ) were added dimethyl phosphite $(46.7 \mu \mathrm{L}$, $0.50 \mathrm{mmol})$ in ethanol $(3.0 \mathrm{~mL})$, then purified by column chromatography ( $\mathrm{SiO}_{2}$, ethyl acetate/hexane $\left.=30-80 \%\right)$ to provide $3 \mathbf{b a}$ as a colorless oil (49.3 mg, 53\% yield). ${ }^{1} \mathrm{H}$ NMR (400 $\left.\mathrm{MHz}, \mathrm{CDCl}_{3}\right): \delta 7.78-7.84(\mathrm{~m}, 2 \mathrm{H}), 7.56-7.60(\mathrm{~m}, 1 \mathrm{H}), 7.46-7.51$ (m, 2H), 3.78 (s, 3H), $3.75(\mathrm{~s}, 3 \mathrm{H}) ;{ }^{13} \mathrm{C} \mathrm{NMR}\left(100 \mathrm{MHz}, \mathrm{CDCl}_{3}\right)$ : $\delta 132.6\left(\mathrm{~d}, J_{\mathrm{C}-\mathrm{P}}=3.0 \mathrm{~Hz}\right), 131.8\left(\mathrm{~d}, J_{\mathrm{C}-\mathrm{P}}=9.9 \mathrm{~Hz}\right), 128.5\left(\mathrm{~d}, J_{\mathrm{C}-\mathrm{P}}=\right.$ $15.2 \mathrm{~Hz}), 126.8\left(\mathrm{~d}, J_{\mathrm{C}-\mathrm{P}}=187.8 \mathrm{~Hz}\right), 52.6\left(\mathrm{~d}, J_{\mathrm{C}-\mathrm{P}}=5.7 \mathrm{~Hz}\right) ;{ }^{31} \mathrm{P}$ NMR (162 MHz, $\left.\mathrm{CDCl}_{3}\right): \delta 22.33$.

Dimethyl 3-methylphenylphosphonate (3bd). Following the general procedure for compound 3, using 3-methylphenylboronic acid pinacol ester (163.6 mg, $0.75 \mathrm{mmol}), \mathrm{Pd}(\mathrm{OAc})_{2}(5.6 \mathrm{mg}$, $5 \mathrm{~mol} \%), \mathrm{Ag}_{2} \mathrm{CO}_{3}(0.21 \mathrm{~g}, 0.75 \mathrm{mmol})$ and tetramethylammonium chloride ( $82 \mathrm{mg}, 0.75 \mathrm{mmol}$ ) were added dimethyl phosphite $(46.7 \mu \mathrm{L}, 0.50 \mathrm{mmol})$ in ethanol $(3.0 \mathrm{~mL})$, then purified by column chromatography $\left(\mathrm{SiO}_{2}\right.$, ethyl acetate/hexane $\left.=30-80 \%\right)$ to provide $3 \mathbf{b d}$ as a colorless oil (48.0 mg, $48 \%$ yield). ${ }^{1} \mathrm{H}$ NMR $\left(400 \mathrm{MHz}, \mathrm{CDCl}_{3}\right): \delta 7.57-7.65(\mathrm{~m}, 2 \mathrm{H}), 7.38(\mathrm{~b}, 2 \mathrm{H}), 3.73-3.78$ $(\mathrm{m}, 6 \mathrm{H}), 2.40(\mathrm{~s}, 3 \mathrm{H}) ;{ }^{13} \mathrm{C} \mathrm{NMR}\left(100 \mathrm{MHz}, \mathrm{CDCl}_{3}\right): \delta 138.4\left(\mathrm{~d}, J_{\mathrm{C}-\mathrm{P}}\right.$ $=15.2 \mathrm{~Hz}), 133.4\left(\mathrm{~d}, J_{\mathrm{C}-\mathrm{P}}=3.1 \mathrm{~Hz}\right), 132.3\left(\mathrm{~d}, J_{\mathrm{C}-\mathrm{P}}=9.8 \mathrm{~Hz}\right), 128.7$ $\left(\mathrm{d}, J_{\mathrm{C}-\mathrm{P}}=39.1 \mathrm{~Hz}\right), 128.6\left(\mathrm{~d}, J_{\mathrm{C}-\mathrm{P}}=45.5 \mathrm{~Hz}\right), 126.5\left(\mathrm{~d}, J_{\mathrm{C}-\mathrm{P}}=\right.$ $186.7 \mathrm{~Hz}), 52.6\left(\mathrm{~d}, J_{\mathrm{C}-\mathrm{P}}=5.3 \mathrm{~Hz}\right), 21.3 ;{ }^{31} \mathrm{P} \mathrm{NMR}(162 \mathrm{MHz}$, $\mathrm{CDCl}_{3}$ ): $\delta$ 22.80; HRMS-EI calcd for $\mathrm{C}_{9} \mathrm{H}_{13} \mathrm{O}_{3} \mathrm{P}[\mathrm{M}]^{+}:$200.0602, found: 200.0597 .

Dibutyl phenylphosphonate (3ca): ${ }^{11}$ Following the general procedure for compound 3, using phenylboronic acid pinacol ester $2 \mathrm{a}(153 \mathrm{mg}, 0.75 \mathrm{mmol}), \mathrm{Pd}(\mathrm{OAc})_{2}(5.6 \mathrm{mg}, 5 \mathrm{~mol} \%)$, $\mathrm{Ag}_{2} \mathrm{CO}_{3}(0.21 \mathrm{~g}, 0.75 \mathrm{mmol})$ and tetramethylammonium chloride $(82 \mathrm{mg}, 0.75 \mathrm{mmol})$ were added dibutyl phosphite (101.5 $\mu \mathrm{L}, 0.50 \mathrm{mmol})$ in ethanol $(3.0 \mathrm{~mL})$, then purified by column chromatography ( $\mathrm{SiO}_{2}$, ethyl acetate/hexane $\left.=20-70 \%\right)$ to provide 3ca as a colorless oil (93.2 mg, 69\% yield). ${ }^{1} \mathrm{H}$ NMR (400 $\left.\mathrm{MHz} \mathrm{CDCl}_{3}\right): \delta 7.77-7.84(\mathrm{~m}, 2 \mathrm{H}), 7.53-7.58(\mathrm{~m}, 1 \mathrm{H}), 7.44-7.49$ (m, 2H), 3.96-4.12 (m, 4H), 1.61-1.69 (m, 4H), 1.34-1.44 (m, $4 \mathrm{H}), 0.90(\mathrm{t}, J=7.2 \mathrm{~Hz}, 6 \mathrm{H}) ;{ }^{13} \mathrm{C} \mathrm{NMR}\left(100 \mathrm{MHz} \mathrm{CDCl}_{3}\right): \delta 132.3$, $131.7\left(\mathrm{~d}, J_{\mathrm{C}-\mathrm{P}}=9.1 \mathrm{~Hz}\right), 128.4\left(\mathrm{~d}, J_{\mathrm{C}-\mathrm{P}}=14.5 \mathrm{~Hz}\right), 128.3\left(\mathrm{~d}, J_{\mathrm{C}-\mathrm{P}}=\right.$ $186.6 \mathrm{~Hz}), 65.7\left(\mathrm{~d}, J_{\mathrm{C}-\mathrm{P}}=5.4 \mathrm{~Hz}\right), 32.4\left(\mathrm{~d}, J_{\mathrm{C}-\mathrm{P}}=6.4 \mathrm{~Hz}\right), 18.7$, 13.5; ${ }^{31} \mathrm{P}$ NMR $\left(162 \mathrm{MHz}, \mathrm{CDCl}_{3}\right): \delta 19.36$. 
Dibutyl $\boldsymbol{p}$-tolylphosphonate (3cb):. ${ }^{15}$ Following the general procedure for compound 3 , using $p$-tolylphenylboronic acid pinacol ester (163.6 mg, $0.75 \mathrm{mmol}), \mathrm{Pd}(\mathrm{OAc})_{2}(5.6 \mathrm{mg}, 5 \mathrm{~mol} \%)$, $\mathrm{Ag}_{2} \mathrm{CO}_{3}(0.21 \mathrm{~g}, 0.75 \mathrm{mmol})$ and tetramethylammonium chloride $(82 \mathrm{mg}, 0.75 \mathrm{mmol}$ ) were added dibutyl phosphite (101.5 $\mu \mathrm{L}, 0.50 \mathrm{mmol})$ in ethanol $(3.0 \mathrm{~mL})$, then purified by column chromatography $\left(\mathrm{SiO}_{2}\right.$, ethyl acetate/hexane $\left.=20-70 \%\right)$ to provide $3 \mathbf{c b}$ as a colorless oil $(86.7 \mathrm{mg}, 61 \%$ yield $) .{ }^{1} \mathrm{H}$ NMR $(400$ $\mathrm{MHz}_{\mathrm{CDCl}}$ ): $\delta 7.72(\mathrm{~d}, J=8.0 \mathrm{~Hz}, 1 \mathrm{H}), 7.68(\mathrm{~d}, J=8.0 \mathrm{~Hz}, 1 \mathrm{H})$, 7.25-7.29 (m, 2H), 3.96-4.09 (m, 4H), 2.40 (s, 3H), 1.65 (quin, $J=$ $6.8 \mathrm{~Hz}, 4 \mathrm{H}), 1.34-1.44(\mathrm{~m}, 4 \mathrm{H}), 0.90(\mathrm{t}, J=7.2,6 \mathrm{H}) ;{ }^{13} \mathrm{C} \mathrm{NMR}$ $\left(100 \mathrm{MHz}, \mathrm{CDCl}_{3}\right): \delta 142.7\left(\mathrm{~d}, J_{\mathrm{C}-\mathrm{P}}=2.3 \mathrm{~Hz}\right), 131.6\left(\mathrm{~d}, J_{\mathrm{C}-\mathrm{P}}=10.2\right.$ $\mathrm{Hz}), 129.0\left(\mathrm{~d}, J_{\mathrm{C}-\mathrm{P}}=15.2 \mathrm{~Hz}\right), 124.7\left(\mathrm{~d}, J_{\mathrm{C}-\mathrm{P}}=189.4 \mathrm{~Hz}\right), 65.5(\mathrm{~d}$, $\left.J_{\mathrm{C}-\mathrm{P}}=5.7 \mathrm{~Hz}\right), 32.2\left(\mathrm{~d}, J_{\mathrm{C}-\mathrm{P}}=6.5 \mathrm{~Hz}\right), 21.4,18.5,13.4 ;{ }^{31} \mathrm{P} \mathrm{NMR}$ $\left(162 \mathrm{MHz}, \mathrm{CDCl}_{3}\right): \delta 20.09$.

Dibutyl 4-methoxyphenylphosphonate (3cc): ${ }^{26}$ Following the general procedure for compound 3, using 4-methoxyphenylboronic acid pinacol ester (175.6 $\mathrm{mg}, 0.75 \mathrm{mmol}$ ), $\mathrm{Pd}(\mathrm{OAc})_{2}(5.6 \mathrm{mg}, 5 \mathrm{~mol} \%), \mathrm{Ag}_{2} \mathrm{CO}_{3}(0.21 \mathrm{~g}, 0.75 \mathrm{mmol})$ and tetramethylammonium chloride $(82 \mathrm{mg}, 0.75 \mathrm{mmol})$ were added dibutyl phosphite $(101.5 \mu \mathrm{L}, 0.50 \mathrm{mmol})$ in ethanol (3.0 $\mathrm{mL})$, then purified by column chromatography $\left(\mathrm{SiO}_{2}\right.$, ethyl acetate/hexane $=20-70 \%)$ to provide $3 \mathbf{c c}$ as a colorless oil (102.1 mg, 68\% yield). ${ }^{1} \mathrm{H}$ NMR (400 MHz, $\left.\mathrm{CDCl}_{3}\right): \delta 7.75$ (d, $J=$ $8.8 \mathrm{~Hz}, 1 \mathrm{H}), 7.72(\mathrm{~d}, J=8.8 \mathrm{~Hz}, 1 \mathrm{H}), 6.95-6.99(\mathrm{~m}, 2 \mathrm{H}), 3.94-4.07$ (m, 4H), 3.85 (s, 3H), 1.60-1.68 (m, 4H), 1.35-1.44 (m, 4H), 0.90 $(\mathrm{t}, J=7.2 \mathrm{~Hz}, 6 \mathrm{H}) ;{ }^{13} \mathrm{C} \mathrm{NMR}\left(100 \mathrm{MHz}, \mathrm{CDCl}_{3}\right): \delta 162.7\left(\mathrm{~d}, J_{\mathrm{C}-\mathrm{P}}=\right.$ $2.8 \mathrm{~Hz}), 133.6\left(\mathrm{~d}, J_{\mathrm{C}-\mathrm{P}}=10.9 \mathrm{~Hz}\right), 119.3\left(\mathrm{~d}, J_{\mathrm{C}-\mathrm{P}}=192.9 \mathrm{~Hz}\right)$, $113.8\left(\mathrm{~d}, J_{\mathrm{C}-\mathrm{P}}=15.4 \mathrm{~Hz}\right), 65.5\left(\mathrm{~d}, J_{\mathrm{C}-\mathrm{P}}=5.4 \mathrm{~Hz}\right), 55.1,32.3\left(\mathrm{~d}, J_{\mathrm{C}-\mathrm{P}}\right.$ $=6.3 \mathrm{~Hz}$ ), 18.6, 13.4; ${ }^{31} \mathrm{P} \mathrm{NMR}\left(162 \mathrm{MHz}, \mathrm{CDCl}_{3}\right): \delta$ 20.47; HRMS-EI calcd for $\mathrm{C}_{15} \mathrm{H}_{25} \mathrm{O}_{4} \mathrm{P}[\mathrm{M}]^{+}: 300.1490$, found: 300.1484 .

Dibutyl 3-methylphenylphosphonate (3cd). Following the general procedure for compound 3, using 3-methylphenylboronic acid pinacol ester $(163.6 \mathrm{mg}, 0.75 \mathrm{mmol}), \mathrm{Pd}(\mathrm{OAc})_{2}(5.6 \mathrm{mg}$, $5 \mathrm{~mol} \%), \mathrm{Ag}_{2} \mathrm{CO}_{3}(0.21 \mathrm{~g}, 0.75 \mathrm{mmol})$ and tetramethylammonium chloride ( $82 \mathrm{mg}, 0.75 \mathrm{mmol}$ ) were added dibutyl phosphite $(101.5 \mu \mathrm{L}, 0.50 \mathrm{mmol})$ in ethanol $(3.0 \mathrm{~mL})$, then purified by column chromatography $\left(\mathrm{SiO}_{2}\right.$, ethyl acetate/hexane $\left.=20-70 \%\right)$ to provide $3 \mathrm{~cd}$ as a colorless oil (78.1 mg, 55\% yield). ${ }^{1} \mathrm{H}$ NMR (400 MHz, $\left.\mathrm{CDCl}_{3}\right): \delta 7.56-7.65(\mathrm{~m}, 2 \mathrm{H}), 7.34-7.37(\mathrm{~m}, 2 \mathrm{H}), 3.95-$ $4.11(\mathrm{~m}, 4 \mathrm{H}), 2.40(\mathrm{~s}, 3 \mathrm{H}), 1.62-1.70(\mathrm{~m}, 4 \mathrm{H}), 1.34-1.45(\mathrm{~m}, 4 \mathrm{H})$, $0.91(\mathrm{t}, J=7.6 \mathrm{~Hz}, 6 \mathrm{H}) ;{ }^{13} \mathrm{C} \mathrm{NMR}\left(100 \mathrm{MHz}, \mathrm{CDCl}_{3}\right): \delta 138.1$ (d, $\left.J_{\mathrm{C}-\mathrm{P}}=14.5 \mathrm{~Hz}\right), 133.0,132.2\left(\mathrm{~d}, J_{\mathrm{C}-\mathrm{P}}=10 \mathrm{~Hz}\right), 128.5\left(\mathrm{~d}, J_{\mathrm{C}-\mathrm{P}}=40\right.$ $\mathrm{Hz}), 128.4\left(\mathrm{~d}, J_{\mathrm{C}-\mathrm{P}}=46.4 \mathrm{~Hz}\right), 128.0\left(\mathrm{~d}, J_{\mathrm{C}-\mathrm{P}}=187.5 \mathrm{~Hz}\right), 65.6(\mathrm{~d}$, $\left.J_{\mathrm{C}-\mathrm{P}}=5.5 \mathrm{~Hz}\right), 32.3\left(\mathrm{~d}, J_{\mathrm{C}-\mathrm{P}}=7.3 \mathrm{~Hz}\right), 21.2,18.6,13.5 ;{ }^{31} \mathrm{P} \mathrm{NMR}$ $\left(162 \mathrm{MHz}, \mathrm{CDCl}_{3}\right): \delta$ 19.91; HRMS-EI calcd for $\mathrm{C}_{15} \mathrm{H}_{25} \mathrm{O}_{3} \mathrm{P}[\mathrm{M}]^{+}$: 284.1541, found: 284.1540 .

Dibutyl 3,5-dimethylphenylphosphonate (3ce). Following the general procedure for compound 3, using 3,5-dimethylphenylboronic acid pinacol ester (174.1 $\mathrm{mg}, 0.75 \mathrm{mmol}$ ), $\mathrm{Pd}(\mathrm{OAc})_{2}$ (5.6 mg, $\left.5 \mathrm{~mol} \%\right), \mathrm{Ag}_{2} \mathrm{CO}_{3}(0.21 \mathrm{~g}, 0.75 \mathrm{mmol})$ and tetramethylammonium chloride $(82 \mathrm{mg}, 0.75 \mathrm{mmol})$ were added dibutyl phosphite $(101.5 \mu \mathrm{L}, 0.50 \mathrm{mmol})$ in ethanol (3.0 $\mathrm{mL})$, then purified by column chromatography $\left(\mathrm{SiO}_{2}\right.$, ethyl acetate/hexane $=20-70 \%)$ to provide 3ce as a colorless oil (99.8 mg, 67\% yield). ${ }^{1} \mathrm{H}$ NMR (400 $\left.\mathrm{MHz} \mathrm{CDCl}_{3}\right): \delta 7.43(\mathrm{~s}, 1 \mathrm{H})$,
7.39 (s, 1H), 7.17 (s, 1H), 3.96-4.09 (m, 4H), 2.35 (s, 6H), 1.62$1.70(\mathrm{~m}, 4 \mathrm{H}), 1.36-1.43(\mathrm{~m}, 4 \mathrm{H}), 0.91(\mathrm{t}, J=7.2,6 \mathrm{H}) ;{ }^{13} \mathrm{C} \mathrm{NMR}$ $\left(100 \mathrm{MHz}, \mathrm{CDCl}_{3}\right): \delta 138.0\left(\mathrm{~d}, J_{\mathrm{C}-\mathrm{P}}=15.4 \mathrm{~Hz}\right), 133.95\left(\mathrm{~d}, J_{\mathrm{C}-\mathrm{P}}=\right.$ $2.7 \mathrm{~Hz}), 129.3\left(\mathrm{~d}, J_{\mathrm{C}-\mathrm{P}}=10 \mathrm{~Hz}\right), 127.8\left(\mathrm{~d}, J_{\mathrm{C}-\mathrm{P}}=184.8 \mathrm{~Hz}\right), 65.6$ $\left(\mathrm{d}, J_{\mathrm{C}-\mathrm{P}}=5.5 \mathrm{~Hz}\right), 32.4\left(\mathrm{~d}, J_{\mathrm{C}-\mathrm{P}}=7.3 \mathrm{~Hz}\right), 21.1,18.6,13.5 ;{ }^{31} \mathrm{P}$ NMR (162 MHz, $\mathrm{CDCl}_{3}$ ): $\delta$ 20.40; HRMS-EI calcd for $\mathrm{C}_{16} \mathrm{H}_{27} \mathrm{O}_{3} \mathrm{P}$ $[\mathrm{M}]^{+}:$298.1698, found: 298.1707 .

Dibutyl (3-methoxy-5-methylphenyl)phosphonate (3cf). Following the general procedure for compound 3, using (3methoxy-5-methyl)phenyl boronic acid pinacol ester $(186.1 \mathrm{mg}$, $0.75 \mathrm{mmol}$ ), $\mathrm{Pd}(\mathrm{OAc})_{2}(5.6 \mathrm{mg}, 5 \mathrm{~mol} \%), \mathrm{Ag}_{2} \mathrm{CO}_{3}(0.21 \mathrm{~g}, 0.75$ $\mathrm{mmol}$ ) and tetramethylammonium chloride $(82 \mathrm{mg}, 0.75 \mathrm{mmol})$ were added dibutyl phosphite $(101.5 \mu \mathrm{L}, 0.50 \mathrm{mmol})$ in ethanol $(3.0 \mathrm{~mL})$, then purified by column chromatography $\left(\mathrm{SiO}_{2}\right.$, ethyl acetate/hexane $=20-70 \%)$ to provide $3 \mathbf{c f}$ as a colorless oil (72.3 mg, 46\% yield). ${ }^{1} \mathrm{H}$ NMR (400 MHz, $\left.\mathrm{CDCl}_{3}\right): \delta 7.10-7.23(\mathrm{~m}$, $2 \mathrm{H}), 6.89(\mathrm{~s}, 1 \mathrm{H}), 3.97-4.10(\mathrm{~m}, 4 \mathrm{H}), 3.82(\mathrm{~s}, 3 \mathrm{H}), 2.37(\mathrm{~s}, 3 \mathrm{H})$, 1.62-1.70 (m, 4H), 1.35-1.45 (m, 4H), $0.91(\mathrm{t}, J=7.2 \mathrm{~Hz}, 6 \mathrm{H}) ;{ }^{13} \mathrm{C}$ NMR (100 MHz, $\left.\mathrm{CDCl}_{3}\right): \delta 159.4\left(\mathrm{~d}, J_{\mathrm{C}-\mathrm{P}}=19.8 \mathrm{~Hz}\right), 139.9\left(\mathrm{~d}, J_{\mathrm{C}-\mathrm{P}}\right.$ $=17.4 \mathrm{~Hz}), 129.0\left(\mathrm{~d}, J_{\mathrm{C}-\mathrm{P}}=185.2 \mathrm{~Hz}\right), 124.7\left(\mathrm{~d}, J_{\mathrm{C}-\mathrm{P}}=9.5 \mathrm{~Hz}\right)$, $119.4\left(\mathrm{~d}, J_{\mathrm{C}-\mathrm{P}}=3.4 \mathrm{~Hz}\right), 113.3\left(\mathrm{~d}, J_{\mathrm{C}-\mathrm{P}}=11 \mathrm{~Hz}\right), 65.75\left(\mathrm{~d}, J_{\mathrm{C}-\mathrm{P}}=\right.$ $5.7 \mathrm{~Hz}), 55.3,32.4\left(\mathrm{~d}, J_{\mathrm{C}-\mathrm{P}}=6.5 \mathrm{~Hz}\right), 21.31\left(\mathrm{~d}, J_{\mathrm{C}-\mathrm{P}}=1.5 \mathrm{~Hz}\right)$, 18.7, 13.5; ${ }^{31} \mathrm{P}$ NMR (162 $\mathrm{MHz}, \mathrm{CDCl}_{3}$ ): $\delta$ 19.69; HRMS-EI calcd for $\mathrm{C}_{16} \mathrm{H}_{27} \mathrm{O}_{4} \mathrm{P}[\mathrm{M}]^{+}$: 314.1647, found: 314.1655.

Dibutyl 3,4-dimethylphenylphosphonate (3cg). Following the general procedure for compound 3, using 3,4-dimethylphenylboronic acid pinacol ester (174.1 $\mathrm{mg}, 0.75 \mathrm{mmol}$ ), $\mathrm{Pd}(\mathrm{OAc})_{2}$ (5.6 mg, $\left.5 \mathrm{~mol} \%\right), \mathrm{Ag}_{2} \mathrm{CO}_{3}(0.21 \mathrm{~g}, 0.75 \mathrm{mmol})$ and tetramethylammonium chloride $(82 \mathrm{mg}, 0.75 \mathrm{mmol}$ ) were added dibutyl phosphite $(101.5 \mu \mathrm{L}, 0.50 \mathrm{mmol})$ in ethanol $(3.0$ $\mathrm{mL})$, then purified by column chromatography $\left(\mathrm{SiO}_{2}\right.$, ethyl acetate/hexane $=20-70 \%$ ) to provide $3 \mathbf{c g}$ as a colorless oil (76.0 mg, 51\% yield). ${ }^{1} \mathrm{H}$ NMR (400 MHz, $\left.\mathrm{CDCl}_{3}\right): \delta 7.49-7.59(\mathrm{~m}$, 2H), 7.20-7.24 (m, 1H), 3.93-4.10 (m, 4H), 2.30 (s, 6H), 1.61-1.69 $(\mathrm{m}, 4 \mathrm{H}), 1.34-1.44(\mathrm{~m}, 4 \mathrm{H}), 0.91(\mathrm{t}, J=7.2 \mathrm{~Hz}, 6 \mathrm{H}) ;{ }^{13} \mathrm{C} \mathrm{NMR}(100$ $\left.\mathrm{MHz}, \mathrm{CDCl}_{3}\right): \delta 141.6,136.9\left(\mathrm{~d}, J_{\mathrm{C}-\mathrm{P}}=15.4 \mathrm{~Hz}\right), 132.8\left(\mathrm{~d}, J_{\mathrm{C}-\mathrm{P}}=\right.$ $10.0 \mathrm{~Hz}), 129.6\left(\mathrm{~d}, J_{\mathrm{C}-\mathrm{P}}=43.7 \mathrm{~Hz}\right), 129.4\left(\mathrm{~d}, J_{\mathrm{C}-\mathrm{P}}=38.2 \mathrm{~Hz}\right)$, $125.2\left(\mathrm{~d}, J_{\mathrm{C}-\mathrm{P}}=188.4 \mathrm{~Hz}\right), 65.6\left(\mathrm{~d}, J_{\mathrm{C}-\mathrm{P}}=6.4 \mathrm{~Hz}\right), 32.4\left(\mathrm{~d}, J_{\mathrm{C}-\mathrm{P}}=\right.$ $7.3 \mathrm{~Hz}), 19.9,19.6,18.7,13.5 ;{ }^{31} \mathrm{P}$ NMR (162 $\left.\mathrm{MHz}, \mathrm{CDCl}_{3}\right)$ : $\delta$ 20.51; HRMS-EI calcd for $\mathrm{C}_{16} \mathrm{H}_{27} \mathrm{O}_{3} \mathrm{P}[\mathrm{M}]^{+}:$298.1698, found: 298.1700 .

\section{Acknowledgements}

The Ministry of Science and Technology, Taiwan (Most 1052113-M-005-001-) and the National Chung Hsing University are gratefully acknowledged for financial support.

\section{References}

1 (a) L. D. Quin, A Guide to Organophosphorus Chemistry, Wiley Interscience, New York, 2000; (b) Organophosphorus Reagents, ed. P. J. Murphy, Oxford University Press, Oxford, U.K., 2004; (c) W. Tang and X. Zhang, Chem. Rev., 2003, 103, 3029-3070; (d) C. Queffélec, M. Petit, P. Janvier, D. A. Knight and B. Bujoli, Chem. Rev., 2012, 112, 3777-3807. 
2 (a) Y. C. Kim, S. G. Brown, T. K. Harden, J. L. Boyer, G. Dubyak, B. F. King, G. Burnstock and K. A. Jacobson, J. Med. Chem., 2001, 44, 340-349; (b) J. J. Shie, J. M. Fang, S. Y. Wang, K. C. Tsai, Y. S. Cheng, A. S. Yang, S. C. Hsiao, C. Y. Su and C. H. Wong, J. Am. Chem. Soc., 2007, 129, 11892-11893; (c) T. S. Kumar, S. Y. Zhou, B. V. Joshi, R. Balasubramanian, T. H. Yang, B. T. Liang and K. A. Jacobson, J. Med. Chem., 2010, 53, 2562-2576.

3 (a) H. H. Chou and C. H. Cheng, Adv. Mater., 2010, 22, 24682471; (b) F. M. Hsu, C. H. Chien, C. F. Shu, C. H. Lai, C. C. Hsieh, K. W. Wang and P. T. Chou, Adv. Funct. Mater., 2009, 19, 2834-2843.

4 (a) T. Baumgartner and R. Reau, Chem. Rev., 2006, 106, 46814727; (b) R. Engel, Chem. Rev., 1977, 77, 349-367.

5 (a) H. A. McManus and P. J. Guiry, Chem. Rev., 2004, 104, 4151-4202; (b) P. W. N. M. van Leeuwen, P. C. J. Kamer, J. N. H. Reek and R. Dierkes, Chem. Rev., 2000, 100, 27412770.

6 (a) T. Hirao, T. Masunaga, Y. Ohshiro and T. Agawa, Tetrahedron Lett., 1980, 21, 3595-3598; (b) T. Hirao, T. Masunaga, Y. Ohshiro and T. Agawa, Synthesis, 1981, 56-57; (c) T. Hirao, T. Masunaga, N. Yamada, Y. Ohshiro and T. Agawa, Bull. Chem. Soc. Jpn., 1982, 55, 909-913.

7 (a) D. Gelman, L. Jiang and S. L. Buchwald, Org. Lett., 2003, 5, 2315-2318; (b) H.-H. Rao, Y. Jin, H. Fu, Y.-Y. Jiang and Y.-F. Zhao, Chem.-Eur. J., 2006, 12, 3636-3646; (c) C. Huang, X. Tang, H. Fu, Y.-Y. Jiang and Y.-F. Zhao, J. Org. Chem., 2006, 71, 5020-5022; (d) M. Kalek, A. Ziadi and J. Stawinski, Org. Lett., 2008, 10, 4637-4640; (e) M. Kalek and J. Stawinski, Organometallics, 2008, 27, 5876-5888; (f) M. Kalek, M. Jezowska and J. Stawinski, Adv. Synth. Catal., 2009, 351, 3207-3206; (g) N. T. McDougal, J. Streuff, H. Mukherjee, S. C. Virgil and B. M. Stoltz, Tetrahedron Lett., 2010, 51, 5550-5554; $(h)$ E. L. Deal, C. Petit and J. L. Montchamp, Org. Lett., 2011, 13, 3270-3273; (i) X.-H. Zhang, H.-Z. Liu, X.-M. Hu, G. Tang, J. Zhu and Y.-F. Zhao, Org. Lett., 2011, 13, 3478-3481; (j) H.-Y. Zhang, M. Sun, Y.-N. Ma, Q.-P. Tiana and S.-D. Yang, Org. Biomol. Chem., 2012, 10, 9627-9633; (k) M. Sun, Y.-S. Zang, L.-K. Hou, X.-X. Chen, W. Sun and S.-D. Yang, Eur. J. Org. Chem., 2014, 6796-6801.

8 (a) K. S. Petrakis and T. L. Nagabhushan, J. Am. Chem. Soc., 1987, 109, 2831-2833; (b) X. Lu and J. Zhu, Synthesis, 1987, 726-727.

9 Y. Luo and J. Wu, Organometallics, 2009, 28, 6823-6826.

10 R. Berrino, S. Cacchi, G. Fabrizi, A. Goggiamani and P. Stabile, Org. Biomol. Chem., 2010, 8, 4518-4520.

11 (a) R. A. Dhokale and S. B. Mhaske, Org. Lett., 2013, 15, 22182221; (b) Q. Chen, X. Yan, Z. Du, K. Zhang and C. Wen, J. Org. Chem., 2016, 81, 276-281; (c) G. Yang, C. Shen, M. Quan and W. Zhang, Tetrahedron, 2016, 72, 333-337.
12 (a) S.-Y. Chen, R.-S. Zeng, J.-P. Zou and O. T. Asekun, J. Org. Chem., 2014, 79, 1449-1453; (b) W. Xu, G. Hu, P. Xu, Y. Gao, Y. Yin and Y. Zhao, Adv. Synth. Catal., 2014, 356, 2948-2954. 13 (a) M. Sun, H.-Y. Zhang, Q. Han, K. Yang and S.-D. Yang, Chem.-Eur. J., 2011, 17, 9566-9570; (b) J.-S. Zhang, T. Chen, J. Yanga and L.-B. Han, Chem. Commun., 2015, 51, 7540-7542.

14 J. Yang, T. Chen and L.-B. Han, J. Am. Chem. Soc., 2015, 137, 1782-1785.

15 T. Miao and L. Wang, Adv. Synth. Catal., 2014, 356, 967-971. 16 J. Xu, P. Zhang, Y. Gao, Y. Chen, G. Tang and Y. Zhao, J. Org. Chem., 2013, 78, 8176-8183.

17 (a) Y. Kuninobu, T. Yoshida and K. Takai, J. Org. Chem., 2011, 76, 7370-7376; (b) C.-G. Feng, M. Ye, K.-J. Xiao, S. Li and J.-Q. Yu, J. Am. Chem. Soc., 2013, 135, 9322-9325; (c) S. Wang, R. Guo, G. Wang, S.-Y. Chen and X.-Q. Yu, Chem. Commun., 2014, 50, 12718-12721.

18 (a) H. C. Brown, G. W. Kramer, A. B. Levy and M. M. Midland, Organic Syntheses via Boranes, Wiley-Interscience, New York, 1975; (b) H. C. Brown and B. Singaram, Acc. Chem. Res., 1988, 21, 287-293; (c) C. M. Crudden and D. Edwards, Eur. J. Org. Chem., 2003, 4695-4712.

19 N. Miyaura and A. Suzuki, Chem. Rev., 1995, 95, 2457-2483. 20 (a) D. M. T. Chan, K. L. Monaco, R. Li, D. Bonne, C. G. Clark and P. Y. S. Lam, Tetrahedron Lett., 2003, 44, 3863-3865; (b) C. C. Tzschucke, J. M. Murphy and J. F. Hartwig, Org. Lett., 2007, 9, 761-764; (c) J.-H. Cheng, C.-L. Yi, T.-J. Liu and C.-F. Lee, Chem. Commun., 2012, 48, 8440-8442; (d) C.-L. Yi, T.-J. Liu, J.-H. Cheng and C.-F. Lee, Eur. J. Org. Chem., 2013, 2013, 3910-3918.

21 (a) M. P. Sibi, H. Tatamidani and K. Patil, Org. Lett., 2005, 7, 2571-2573; (b) M. Ogasawara, H. L. Ngo, T. Sakamoto, T. Takahashi and W. Lin, Org. Lett., 2005, 7, 2881-2884; (c) G. Chen, N. Tokunaga and T. Hayashi, Org. Lett., 2005, 7, 2285-2288.

22 (a) M. Andaloussi, J. Lindh, J. Savmarker, P. J. R. Sjoberg and M. Larhed, Chem.-Eur. J., 2009, 15, 13069-13074; (b) R.-Q. Zhuang, J. Xu, Z.-S. Cai, G. Tang, M.-J. Fang and Y.-F. Zhao, Org. Lett., 2011, 13, 2110-2113; (c) G. Hu, W. Chen, T. Fu, Z. Peng, H. Qiao, Y. Gao and Y. Zhao, Org. Lett., 2013, 15, 5362-5365; (d) T. Fu, H. Qiao, Z. Peng, G. $\mathrm{Hu}, \mathrm{X} . \mathrm{Wu}, \mathrm{Y}$. Gao and Y. Zhao, Org. Biomol. Chem., 2014, 12, 2895-2902.

23 M. C. Kohler, T. V. Grimes, X. Wang, T. R. Cundari and R. A. Stockland Jr, Organometallics, 2009, 28, 1193-1201.

24 J. Ratniyom, N. Dechnarong, S. Yotphan and S. Kiatisevi, Eur. J. Org. Chem., 2014, 2014, 1381-1385.

25 B. M. Partridge and J. F. Hartwig, Org. Lett., 2013, 15, 140143.

26 X. Lu and J. Zhu, Synthesis, 1987, 726-727. 